\section{ORIGINAL RESEARCH}

M.-S. Ko

K.S. Jeong

Y.K. Shong

G.Y. Gong

J.H. Baek

J.H. Lee

\title{
Collapsing Benign Cystic Nodules of the Thyroid Gland: Sonographic Differentiation from Papillary Thyroid Carcinoma
}

\begin{abstract}
BACKGROUND AND PURPOSE: The US features of benign and malignant nodules overlap, and benign thyroid lesions can mimic thyroid malignancy on US. Benign cystic nodules after spontaneous collapse or needle aspiration, can mimic malignant thyroid nodules. Our aim was to evaluate the US features of $\mathrm{CBCNs}$ of the thyroid that distinguish such nodules from malignant thyroid nodules.
\end{abstract}

\begin{abstract}
MATERIALS AND METHODS: US and clinical findings in 13 patients, each with a single CBCN, were evaluated to determine if they showed $>50 \%$ cystic content on initial US or CT and $>30 \%$ decrease in maximum diameter on follow-up US. We compared these findings with those of 26 patients, each with a single surgically confirmed PTMC. US scans were analyzed for internal content, shape, margin, echogenicity, presence of echogenic dots suggesting micro- and macrocalcification, inner isoechoic rim, and low-echoic halo.
\end{abstract}

\begin{abstract}
RESULTS: Six of the $13(46 \%)$ CBCNs were classified as malignant on US due to their marked hypoechogenicity, microcalcification, or spiculated margins. US features that differed between CBCNs and PTMCs were shape (ovoid-to-round versus taller-than-wide, $P=.016$ ); margins (ill-defined versus spiculated, $P<.000)$; low-echoic halo $(P<.000)$; inner isoechoic $\operatorname{rim}(P<.000)$ with high negative predictive values $(100 \%, 91 \%, 91 \%$, and $89 \%$, respectively); and clinically acceptable diagnostic accuracy $(59 \%, 80 \%, 82 \%$, and $85 \%$, respectively).
\end{abstract}

CONCLUSIONS: US features helpful for differential diagnosis of CBCNs from PTMCs include shape, margin, and the presence of an inner isoechoic rim and a low-echoic halo. Familiarity with US features suggesting $\mathrm{CBCNs}$ may be helpful in reducing unnecessary repeated FNABs.

ABBREVIATIONS: $\mathrm{CBCN}=$ collapsing benign cystic nodule; FNAB = fine needle aspiration biopsy PTMC = papillary thyroid microcarcinoma; US = sonography

$\mathbf{H}^{\mathrm{i}}$ gh-resolution US has led to the identification of many nodules in the thyroid. ${ }^{1}$ Most of these incidental thyroid nodules are benign; only a small percentage $(9.2 \%-14.8 \%)$ are malignant. $^{2-4}$ US features predictive of malignant nodules include a taller-than-wide shape, irregular or spiculated margins, marked hypoechogenicity, and the presence of micro- or macrocalcifications. ${ }^{2-8}$ Purely cystic nodules, predominantly cystic nodules with comet-tail artifacts and spongiform appearance, are US features predictive of benign thyroid nodules. ${ }^{8}$

The US features of benign and malignant nodules sometimes overlap, and benign thyroid lesions can mimic thyroid malignancy on US. This similarity, in turn, can lead to unnecessary biopsies. CBCNs, which occur either spontaneously or after needle aspiration, can mimic malignant thyroid nodules. ${ }^{9}$ The US features of CBCNs that differentiate them from malignant thyroid nodules have not yet been determined and, we therefore, sought to evaluate these.

Received December 27, 2010; accepted after revision April 23, 2011.

From the Departments of Radiology and Research Institute of Radiology (M.-S.K., J.H.B., J.H.L.), Endocrinology (Y.K.S.), and Pathology (G.Y.G.), University of Ulsan College of Medicine, Asan Medical Center, Seoul, Korea; and Department of Diagnostic Radiology (K.S.J.), College of Medicine, Kosin University, Busan, Korea.

Please address correspondence to Jeong Hyun Lee, MD, Department of Radiology and Research Institute of Radiology Asan Medical Center, 388-1 Poongnap-2dong, Songpa-gu, Seoul, 138-736, Korea; e-mail: jeonghlee@hanmir.com

http://dx.doi.org/10.3174/ajnr.A2732

\section{Materials and Methods}

\section{Patient Selection}

This retrospective study was approved by our institutional review board, and informed-consent documents were waived. We reviewed the computerized medical records and US images of the 2529 patients who underwent thyroid US in our institution from January to June 2007. CBCN was diagnosed when nodules showed $>50 \%$ cystic content on initial US or CT and there was a $>30 \%$ decrease in the long diameter of the initial nodule on follow-up US. We identified 13 patients, each with a single CBCN. During a mean follow-up period of 13 months (range, 3-34 months), 4 of the 13 CBCNs showed $>50 \%$ decreases in the long diameter without pathologic confirmation. The 9 other CBCNs were histologically diagnosed as benign follicular cell lesions $(n=4)$, cystic change with macrophages $(n=4)$, and colloid cyst $(n=1)$ (Table 1$)$. Mean patient age was 54 years, with a range of 39-68 years, and the mean diameters of the $\mathrm{CBCNs}$ on initial and follow-up images were $1.4 \mathrm{~cm}$ (range, $0.7-5.0 \mathrm{~cm}$ ) and $0.5 \mathrm{~cm}$ (range, $0-0.9 \mathrm{~cm})$, respectively

For each patient with a $\mathrm{CBCN}$, we also enrolled patients who underwent US and surgery during the same period for PTMC of similar nodule size range. Of the 427 patients who had surgically confirmed PTMCs, we recruited 26 consecutive patients, each with a single PTMC. The mean age of these patients was 42 years (range, 22-63 years), and the mean diameter of the PTMCs was $0.7 \mathrm{~cm}$ (range, $0.3-1.0 \mathrm{~cm}$ ).

\section{Analysis of US Findings and Statistics}

US examinations were performed with an HDI 5000 scanner (PhilipsAdvanced Technology Laboratories, Bothell, Washington) by using 


\begin{tabular}{|c|c|c|c|c|c|c|}
\hline $\begin{array}{l}\text { Case } \\
\text { No. }\end{array}$ & $\begin{array}{l}\text { Age } \\
\text { (yr) }\end{array}$ & Sex & $\begin{array}{c}\mathrm{F} / \mathrm{U} \\
\text { Interval } \\
(\mathrm{mo})\end{array}$ & $\begin{array}{c}\text { Initial } \\
\text { Diameter } \\
\text { (cm) }\end{array}$ & $\begin{array}{c}\mathrm{F} / \mathrm{U} \\
\text { Diameter } \\
(\mathrm{cm})\end{array}$ & Cytology \\
\hline 1 & 64 & $\mathrm{~F}$ & 13 & 0.7 & 0.3 & Cystic change \\
\hline 2 & 59 & $\mathrm{~F}$ & 3 & 1.1 & 0.7 & Cystic change \\
\hline 3 & 60 & $\mathrm{~F}$ & 16 & 5.0 & 0.8 & \\
\hline 4 & 51 & $\mathrm{~F}$ & 6 & 2.4 & 0.4 & \\
\hline 5 & 41 & $\mathrm{~F}$ & 7 & 0.8 & 0.4 & Cystic change \\
\hline 6 & 61 & $\mathrm{~F}$ & 7 & 1.2 & 0.8 & Colloid cyst \\
\hline 7 & 54 & $M$ & 21 & 1.3 & 0.9 & Cystic change \\
\hline 8 & 47 & $\mathrm{~F}$ & 34 & 0.9 & 0.5 & Benign follicular cel \\
\hline 9 & 56 & $M$ & 10 & 0.8 & 0 & \\
\hline 10 & 59 & $\mathrm{~F}$ & 14 & 1.1 & 0.5 & Benign follicular ce \\
\hline 11 & 68 & $M$ & 7 & 1.7 & 0.6 & \\
\hline 12 & 39 & $\mathrm{~F}$ & 21 & 0.8 & 0.3 & Cystic change \\
\hline 13 & 46 & $\mathrm{~F}$ & 25 & 0.7 & 0.4 & Benign follicular ce \\
\hline Mean & 54.2 & & 14.2 & 1.4 & 0.5 & \\
\hline
\end{tabular}

Note:-F/U indicates follow-up.

electronically focused near-field probes with a bandwidth of 7-12 $\mathrm{MHz}$. All US examinations were performed by radiologists with $4-7$ years of experience.

US images were retrospectively reviewed independently by 2 experienced radiologists (K.S.J. and J.H.L., with 7 and 13 years, respectively, of clinical experience in performing and evaluating thyroid US). Both reviewers had no information on patient clinical history, previous imaging results, or pathologic results. Any discrepancies between the 2 reviewers were resolved by consensus.

Individual US features evaluated included nodule shape (oval-toround, irregular, or taller-than-wide), margins (well-defined, ill-defined, or spiculated), internal content (solid, predominantly solid, predominantly cystic, or cystic), and echogenicity (markedly hypoechoic, hypoechoic, isoechoic, or hyperechoic). US results were also evaluated for the presence of echogenic dots suggesting microcalcification, macrocalcification, inner isoechoic rim, and low echoic halo. An inner isoechoic rim was defined as a thin continuous isoechoic rim along more than half the inner margin of a nodule (Fig 1). A low-echoic halo was defined as a hypoechoic rim surrounding the outer margin of a nodule. All other US features were based on previously published criteria. ${ }^{2,5-8,10}$

Statistical analysis was performed by using the Statistical Package for the Social Sciences software, Version 12.0 for Windows (SPSS, Chicago, Illinois). Each of the US features was analyzed for its association with $\mathrm{CBCNs}$ versus malignant nodules. The relevant features were compared with the final diagnosis to calculate the sensitivity, specificity, positive and negative predictive values, and overall diagnostic accuracy. US findings in the $\mathrm{CBCN}$ and PTMC groups were compared by using the Fisher exact test, and nodule size was compared by using the Mann-Whitney $U$ test. A $P$ value $<.05$ was considered statistically significant.

\section{Results}

Demographic data, including age and sex, did not differ significantly between the CBCN and PTMC groups $(P>.05)$. The contents of all $13 \mathrm{CBCNs}$ showed a solid appearance on US, and all 13 had an ovoid-to-round shape and were hypo- or marked hypoechogenic. In addition, $11 \mathrm{CBCNs}$ had ill-defined margins and inner isoechoic rims, while 6 had echogenic dots suggesting microcalcification and 10 had low-echoic haloes (Fig 2).

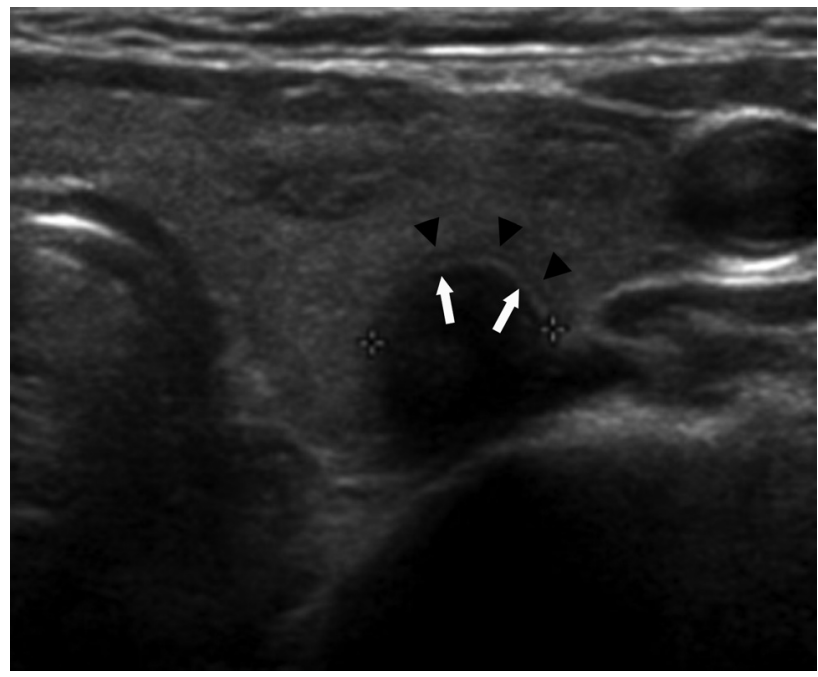

Fig 1. A 59-year-old woman with a CBCN. A routine transverse US shows a 0.8 -cm-long round hypoechoic nodule, with a peripheral low-echoic halo (arrowheads) and an inner isoechoic rim (arrows). Subsequent pathologic examination after surgery confirmed degenerating nodular hyperplasia in the left thyroid gland and papillary carcinoma in the right thyroid gland (not shown).

In comparison, the contents of all 26 PTMCs had a solid appearance on US. All were hypo- or marked hypoechogenic, and 20 had spiculated margins. Microcalcification was observed in 15 patients, and macrocalcification, in 23. Five PTMCs had an inner isoechoic rim, and 3 had low-echoic haloes (Fig 3). Although their incidence rates were low, tallerthan-wide shape and macrocalcification were observed only in PTMCs.

Among the US features, shape, margin, inner isoechoic rim, and low-echoic halo differed significantly between CBCNs and PTMCs (Table 2). The overall diagnostic accuracies of ovoid-to-round shape, ill-defined margins, inner isoechoic rim, and low-echoic halo in differentiating CBCNs from PTMCs were $59 \%, 80 \%, 82 \%$, and $85 \%$, respectively (Table 3).

\section{Discussion}

Several studies have assessed the natural history of thyroid nodules, ${ }^{11,12}$ with $1^{13}$ reporting that the most common outcome of benign thyroid nodules, untreated for an average of 15 years after the first examination, was a decrease in nodule size, including disappearance (52.9\%), with a decrease in size being more prominent in cystic nodules. In contrast, others have reported that $39 \%$ of benign thyroid nodules increase in size during follow-up, with cystic nodules being more likely to maintain or decrease in size than solid nodules. ${ }^{11}$

The revised guidelines of the American Thyroid Association in 2009 recommended that FNAB is warranted for subcentimeter nodules with suspicious findings or in patients at high risk, including patients exposed to radiation or those with a family history of papillary thyroid carcinoma. ${ }^{14}$ Mazzaferri and Sipos have recommended, however, that nodules $<5 \mathrm{~mm}$ should not be biopsied because of high rates of false-positive results on US and of inadequate cytology. ${ }^{15}$ Recently, Moon et $\mathrm{al}^{8}$ reported that US features have been found to depend on the size of thyroid cancers, with subcentimeter tumors having a lower frequency of microcalcification but higher frequencies 


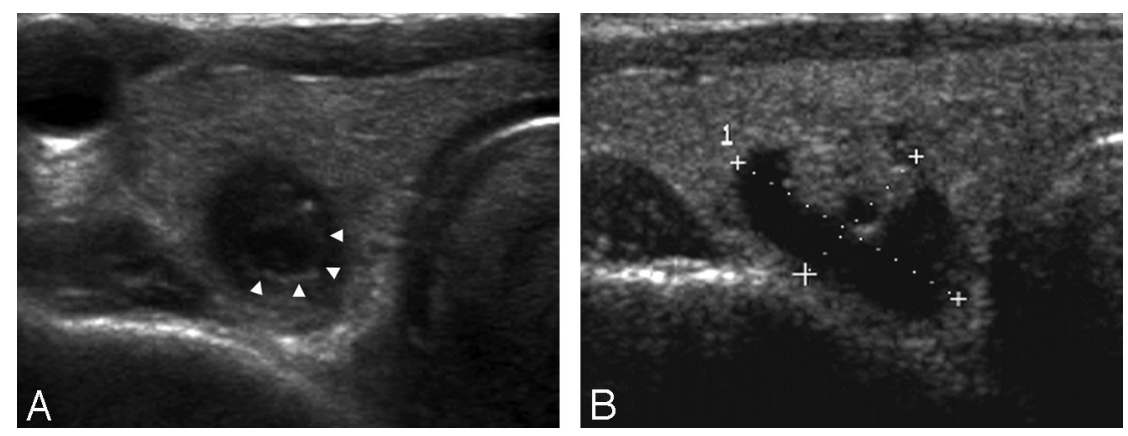

Fig 2. A 68-year-old man with a collapsing benign cystic nodule. A, A transverse US of the thyroid gland shows a 0.6-cm-long ill-defined ovoid markedly hypoechoic nodule with suspicious microcalcification. Note a low-echoic halo and an inner isoechoic rim (arrowheads) surrounding the nodule. B, A transverse US performed 1 year ago reveals a 1.7-cm-long predominantly cystic nodule with an isoechoic solid component at the same location.

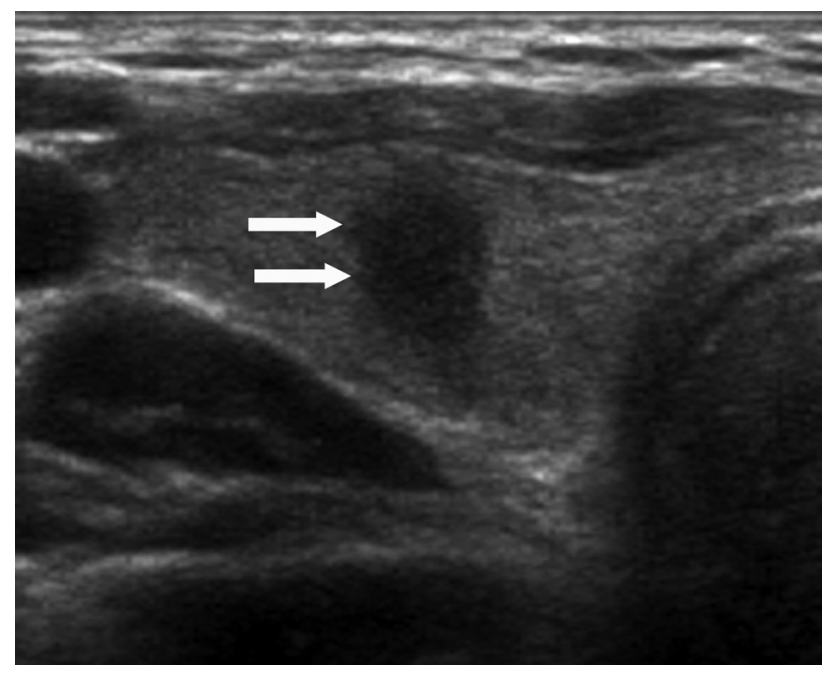

Fig 3. A 42-year-old woman with papillary thyroid carcinoma. Transverse sonogram shows a 0.6-cm-long spiculated taller-than-wide hypoechoic nodule (arrows) within the right lobe of the thyroid gland, which is suspicious for malignancy by US criteria. Note there is neither a low-echoic halo nor an inner isoechoic rim at the periphery. Subsequent cytologic and histologic examination confirmed the diagnosis of papillary thyroid carcinoma.

\begin{tabular}{|c|c|c|c|}
\hline Characteristic & $\operatorname{CBCN}(n=13)$ & PTMC $(n=26)$ & $P$ Value \\
\hline \multicolumn{4}{|l|}{ Internal content } \\
\hline Solid & $13(100)$ & $26(100)$ & \\
\hline Shape & & & .016 \\
\hline Ovoid-to-round & $13(100)$ & $16(62)$ & \\
\hline Taller-than-wide & & $10(39)$ & \\
\hline Margin & & & $<.000$ \\
\hline Spiculated & $2(15)$ & $20(76)$ & \\
\hline III-defined & $11(85)$ & $6(23)$ & \\
\hline Echogenicity & & & .290 \\
\hline Marked hypoechogenicity & $6(46)$ & $7(26.9)$ & \\
\hline Hypoechogenicity & $7(54)$ & $19(73)$ & \\
\hline Microcalcification & $6(46)$ & $15(58)$ & .734 \\
\hline Macrocalcification & $0(0)$ & $23(89)$ & .538 \\
\hline Inner isoechoic rim & $11(85)$ & $5(19)$ & $<.000$ \\
\hline Low-echoic halo & $10(77)$ & $3(1)$ & $<.000$ \\
\hline
\end{tabular}

${ }^{\text {a }}$ Data are numbers of nodules, with percentages in parentheses.

of marked hypoechogenicity, taller-than-wide shape, and spiculated margin. Because these characteristics are also more frequent in smaller benign nodules, the false-positive rate of malignancy may be higher for smaller nodules.

Cytologically diagnosed benign thyroid nodules after aspi-
Table 3: Diagnostic sensitivity, specificity, PPV, NPV, and accuracy of the individual US features suggesting $\mathrm{CBCNs}^{\mathrm{a}}$

\begin{tabular}{lcrrrrr}
\hline Characteristics & $\begin{array}{l}\text { No. } \\
(\%)\end{array}$ & Sensitivity & Specificity & PPV & NPV & Accuracy \\
\hline Ovoid-to-round & $13(100)$ & 100 & 39 & 45 & 100 & 59 \\
III-defined margin & $11(85)$ & 85 & 77 & 65 & 91 & 80 \\
Inner isoechoic rim & $11(85)$ & 85 & 81 & 69 & 91 & 82 \\
Low-echoic halo & $10(77)$ & 77 & 89 & 77 & 89 & 85 \\
\hline
\end{tabular}

Note:-PPV indicates positive predictive value; NPV, negative predictive value. a Data are percentages.

ration may have US features similar to those of malignant thyroid nodules, ${ }^{9,16}$ including marked hypoechogenicity due to solid-appearing internal content. Of the $13 \mathrm{CBCNs}$ that were examined in this study, 6 (46\%) were classified as malignant by US criteria. These US features may be due to collapse of the cystic cavity, infarction of the solid component, and other destructive processes such as hemorrhage, followed by replacement by fibrous tissue. ${ }^{17}$ These pathologic alterations may appear on US as marked hypoechogenicity of a nodule with ill-defined margins.

Other than marked hypoechogenicity of solid-appearing internal content, we found that the US features of $\mathrm{CBCNs}$ that differed significantly from PTMCs were ovoid-to-round shape, ill-defined margins, microcalcification, inner isoechoic rim, and low-echoic halo. Of these US features, inner isoechoic rim and low-echoic halo showed the highest diagnostic accuracies with acceptable negative predictive values. Inner isoechoic rim was the US feature that was first assessed in this study. We defined "inner isoechoic rim" as a thin continuous isoechoic rim present along $>50 \%$ of the inner margin of a thyroid nodule. The pathologic correlate of this is unclear; it may correspond to intact still-solid components at the periphery of a nodule, which appear as isoechoic rims lining the inner wall of pre-existing cystic nodules. Low-echoic halo, a hypoechoic rim surrounding the outer margin of a nodule, can represent a tumor capsule, a fibrotic pseudocapsule, compressed normal thyroid tissue, or chronic inflammatory infiltrates. ${ }^{16,18}$ These features may be common to benign lesions, ${ }^{19-21}$ a finding supported by our results.

Our study had several limitations, including its retrospective design and the small number of patients, which may have introduced a selection bias. However, because the purpose of this study was not to assess the general US features of CBCNs but to determine the specific US features that distinguish CBCNs from PTMCs, this bias may not have had a large im- 
pact on our results. Our results, however, suggest the need for larger scale prospective studies.

\section{Conclusions}

We found that $46 \%$ of CBCNs could be misclassified as malignant on US because they showed marked hypoechogenicity, microcalcification, and/or spiculated margins. Among the US features that can be used to distinguish CBCNs and PTMCs are the presence of an inner isoechoic rim and low-echoic halo, nodule shape, and margin. Therefore, although US features suggesting $\mathrm{CBCNs}$ may not completely abrogate the need for FNAB, familiarity with the US findings of CBCNs may reduce the incidence of unnecessary repeated FNABs. Furthermore, rather than performing immediate FNAB, nodules with US features suggesting $\mathrm{CBCN}$ can be followed-up, especially in patients without a high likelihood of malignancy.

Disclosures: Kyung Soon Jeong, Research Support (including provision of equipment or materials): Kosin University.

\section{References}

1. Ezzat S, Sarti DA, Cain DR, et al. Thyroid incidentalomas. Prevalence by palpation and ultrasonography. Arch Intern Med 1994;154:1838-40

2. Papini E, Guglielmi R, Bianchini A, et al. Risk of malignancy in nonpalpable thyroid nodules: predictive value of ultrasound and color-Doppler features. J Clin Endocrinol Metab 2002;87:1941-46

3. Frates MC, Benson CB, Doubilet PM, et al. Prevalence and distribution of carcinoma in patients with solitary and multiple thyroid nodules on sonography. J Clin Endocrinol Metab 2006;91:3411-17

4. Nam-Goong IS, Kim HY, Gong G, et al. Ultrasonography-guided fine-needle aspiration of thyroid incidentaloma: correlation with pathological findings. Clin Endocrinol (Oxf) 2004;60:21-28

5. Kim EK, Park CS, Chung WY, et al. New sonographic criteria for recommending fine-needle aspiration biopsy of nonpalpable solid nodules of the thyroid. AJR Am J Roentgenol 2002;178:687-91
6. Frates MC, Benson CB, Doubilet PM, et al. Can color Doppler sonography aid in the prediction of malignancy of thyroid nodules? J Ultrasound Med 2003;22: 127-31, quiz 132-34

7. Iannuccilli JD, Cronan JJ, Monchik JM. Risk for malignancy of thyroid nodules as assessed by sonographic criteria: the need for biopsy. J Ultrasound Med 2004;23:1455-64

8. Moon WJ, Jung SL, Lee JH, et al. Benign and malignant thyroid nodules: US differentiation-multicenter retrospective study. Radiology 2008;247:762-70

9. Shin JH, Han BK, Ko EY, et al. Imaging-pathology discordant thyroid nodules: analysis of causes on ultrasonography. J Korean Thyroid Assoc 2008;1:112-16

10. Silver RJ, Parangi S. Management of thyroid incidentalomas. Surg Clin North Am 2004;84:907-19

11. Alexander EK, Hurwitz S, Heering JP, et al. Natural history of benign solid and cystic thyroid nodules. Ann Intern Med 2003;138:315-18

12. Kuma K, Matsuzuka F, Kobayashi A, et al. Outcome of long-standing solitary thyroid nodules. World J Surg 1992;16:583-87, discussion 587-88

13. Kuma K, Matsuzuka F, Yokozawa T, et al. Fate of untreated benign thyroid nodules: results of long-term follow-up. World J Surg 1994;18:495-98, discussion 499

14. Cooper DS, Doherty GM, Haugen BR, et al. Revised American Thyroid Association management guidelines for patients with thyroid nodules and differentiated thyroid cancer. Thyroid 2009;19:1167-214

15. Mazzaferri EL, Sipos J. Should all patients with subcentimeter thyroid nodules undergo fine-needle aspiration biopsy and preoperative neck ultrasonography to define the extent of tumor invasion? Thyroid 2008;18:597-602

16. Koo JH, Shin JH, Han BK, et al. Cystic thyroid nodules after aspiration mimicking malignancy: sonographic characteristics. J Ultrasound Med 2010;29:1415-21

17. Chow SM, Chan JK, Law SC, et al. Diffuse sclerosing variant of papillary thyroid carcinoma: clinical features and outcome. Eur J Surg Oncol 2003;29:446-49

18. Propper RA, Skolnick ML, Weinstein BJ, et al. The nonspecificity of the thyroid halo sign. J Clin Ultrasound 1980;8:129-32

19. Liu FH, Hsueh C, Chang HY, et al. Sonography and fine-needle aspiration biopsy in the diagnosis of benign versus malignant nodules in patients with autoimmune thyroiditis. J Clin Ultrasound 2009;37:487-92

20. Anderson L, Middleton WD, Teefey SA, et al. Hashimoto thyroiditis: Part 2. Sonographic analysis of benign and malignant nodules in patients with diffuse Hashimoto thyroiditis. AJR Am J Roentgenol 2010;195:216-22

21. Kim DW, Lee EJ, Kim SH, et al. Ultrasound-guided fine-needle aspiration biopsy of thyroid nodules: comparison in efficacy according to nodule size. Thyroid 2009;19:27-31 